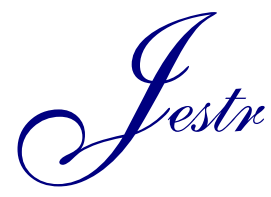

\title{
Optimal PPM-Shift Based on Artificial Neural Network for 2PPM-TH-UWB systems
}

\author{
Abdelaziz Maaref* and Sid Ahmed Elahmar \\ Department of Electronics, Djillali Liabes University, Sidi Bel Abbes, 22000, Algeria
}

Received 24 July 2018; Accepted 14 September 2019

\begin{abstract}
The optimal value of the PPM-Shift related with the binary TH-PPM should be cautiously selected to make sure the finest performance of the PPM-TH-UWB system for a given pulse shape utilized in a specific submission. In addition, it has been demonstrated that the ideal choice of the PPM-Shift parameter denotes back to the autocorrelation characteristics of the pulse shape under examination. In this paper, artificial neural network (ANN) with Levenberg-Marquardt Learning Algorithm (LM) is proposed to optimize PPM-shift for the TH-UWB under Standard Gaussian Approximation (SGA) with multi-Rate system, this optimal PPM-Shift is a function of the data bit Rate $(\mathrm{Rb})$ and Gaussian pulse and its derivatives. Theoretical analysis and simulation results show that our algorithm rapidly converges to the optimal PPMShift and has the advantages of low bit-error rate.
\end{abstract}

Keywords: TH-UWB, Gaussian pulse, PPM, ANN, Levenberg-Marquardt

\section{Introduction}

Ultra-wideband (UWB) technology which uses narrow pulses having duration on the order of nanosecond was approved by the federal communications commission (FCC) in February 2002 for short-range, high speed wireless systems. To avoid interference with the co-existing applications, the radiated power should not exceed -41 $\mathrm{dBm} / \mathrm{MHz}$ within the band from $3.1 \mathrm{GHz}$ to $10.6 \mathrm{GHz}$ [1], In IEEE 802.15.3a and IEEE 802.15.4a UWB is a possible radio technique at physical layer. Because of wideband nature of UWB signal it provides the high data rate in Wireless Personal Area Networks (WPANs) for multimedia traffic. UWB is also known as wireless USB, as it is seen as a replacement of USB cable. In UWB, there are two approaches for multiple accesses. One is based on time hopping (TH), which is known as an Impulse radio [2] and second is based on Multi band OFDM [2].

Depending upon modulation scheme TH UWB signal is known as TH-PPM UWB [2-3-4]. Also Direct Sequence UWB. In all TH-UWB method single bit duration (Tb) is divided into Ns number of frames, each with equal duration of (Ts ) such that, $\mathrm{Tb}=$ NsTs. Further each frame duration (Ts) is divided into Nc number of chips of duration (Tc). During each hip period (Tc) UWB radio signal is transmitted. This UWB pulse is Gaussian pulse or its derivative, which is transmitted depending upon $\mathrm{TH}$ code. UWB radio signal comprised of a sequence of sub-nano second duration

The proposed TH-UWB structure can also achieve variable data Rate transmission or multi service Rate.The Autocorrelation method was used to obtain the optimal PPM-Shift. Its involves a long research and selecting among a wide range of PPM-Shift. It is a time-consuming process and not convenient in practice. In fact, in real-time communication systems, the parameters often change during communication, so the value of the PPM-Shift must be updated accordingly. to address this problem, ANNs are used to find the optimal PPM-Shift. It needs a training sequence to train the network, at a first stage. After that, the model will be autonomous and directly gives the required optimal PPM-Shift. To the best of our knowledge, the ANN is used for the first time, in this paper, to find an adaptive optimal PPM-Shift for a 2PPM-TH-UWB.

\section{Pulse construction for UWB transmitters}

By far the most popular pulse shapes discussed in UWB communication literature are the Gaussian pulse and its derivatives, the basic Gaussian pulse is given as [5-6].

$$
P^{(0)}(t)=\frac{1}{\sqrt{2 \pi \sigma^{2}}} e^{\frac{-(t)^{2}}{2 \sigma^{2}}}
$$

The first and second order Gaussian pulses can be obtained by differentiating $P^{(0)}(t)$ against $t$ as:

$$
P^{(n)}(t)=\frac{d^{n}}{d^{n}(t)} P^{(0)}(t)
$$

Figure 1 show the second derivative Gaussian pulse in the special case of tau $=0.2 \mathrm{~ns}$, tau $=3 \mathrm{~ns}$, and tau $=0.4 \mathrm{~ns}$. 


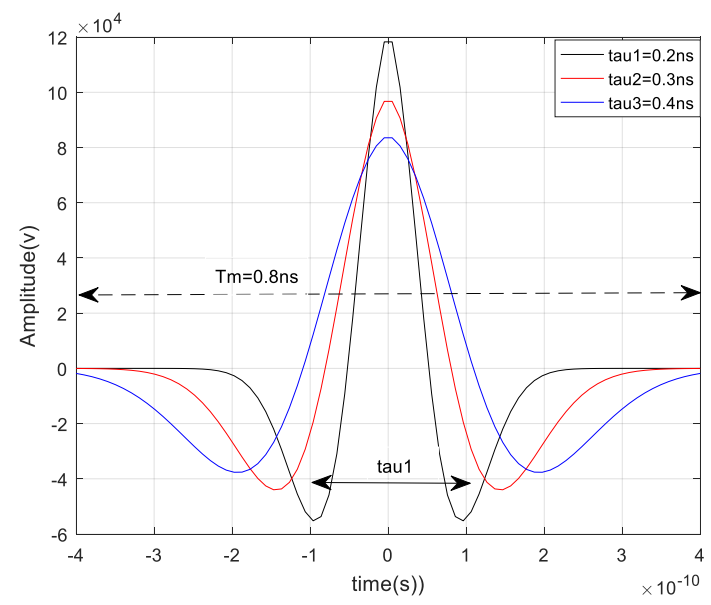

Fig. 1. Second derivative Gaussian pulse in time domain.

\section{Analyzing multiple-user interference}

Our system is the combination of TH code and PPM modulation The transmitted signal of $\mathrm{n}^{\text {th }}$ transmitter is represented as follow [2-3-4-7]:

$s_{T X}^{(n)}=\sum_{j=-\infty}^{\infty} b_{j}^{n} \sqrt{E_{T X}^{(n)}} p_{0}\left(t-j T_{s}-c_{j}^{(n)} T_{c}-a_{j}^{(n)} \varepsilon\right)$

where $p_{0}(t)$ is the energy normalized pulse waveform with duration $T_{M}$ and $E_{T X}^{(n)}$ is the energy transmitted over each single pulse for the $n^{T h}$ device; $C_{j}^{(n)} T_{c}$ is the time shift introduced by the TH code with $C_{j}^{(n)}$ being the $\mathrm{j}$-th pseudorandom code of device $n, T c$ the chip duration and Ts is the average pulse repetition time. The $\mathrm{TH}$ sequence is considered to be a set of independent and identically distributed random variables. The term $a_{j}^{(n)} \varepsilon$ represents the PPM-Shift introduced by the modulation accounting with the number of pulses per bit (Ns). For the binary PPM modulation, $a_{j}^{(n)}$ can assume the binary values $(0$ or 1$)$, the correct detection in the receiver may be easily obtained. The output (Ns, 1) of the code repetition coder receives as input the binary source sequence. With the use of the repetition coder, the average pulse repetition period is considered to be:

$T_{S}=T_{b} / N S$

where

$\mathrm{Tb}$ is the bit time.

Assume that we use IEEE 802.15.3a channel model, and the channel is impacted by thermal noise (Gaussian White Noise), the received signal is the sum of the transmitted signal which from transmitters and the Gaussian White Noise, it can be expressed as [2-3]:

$$
r(t)=\sum_{n=1}^{N_{u}} \sum_{j-\infty}^{\infty} \sqrt{E_{R X}^{(n)}} p_{0}\left(t-j T_{s}-C_{j}^{(n)} T_{c}-a_{j}^{(n)} \varepsilon-\tau^{(n)}\right)+n(t)
$$

The received signal can be defined as, [2-3]:

$r(t)=r_{u}(t)+r_{m u i}(t)+n(t)$

Where received signal contributions are independent $r_{u}(t)$ and $r_{\text {mui }}(t)$ are the useful signal and multiple access interference gathered at the receiver, respectively.

$E_{R X}^{(n)}$ is the energy received over each single pulse and $\mathrm{n}(\mathrm{t})$ is the AWGN noise with double sided spectral density $\mathrm{N} 0 / 2$. The useful received signal is expressed by:

$r(t)_{U}=\sum_{j=0}^{N s-1} \sqrt{E_{R X}^{(1)}} p_{0}\left(t-j T_{s}-C_{j}^{(1)} T_{c}-a_{j}^{(1)} \varepsilon-\tau^{(1)}\right)$

Where $E_{R X}^{(1)}$ is the energy received from a single pulse from the reference transmitter. The multiple access interference signals are expressed by:

$$
r(t)=\sum_{n=2}^{N u} \sum_{j-\infty}^{\infty} \sqrt{E_{R X}^{(n)}} p_{0}\left(t-j T_{s}-C_{j}^{(n)} T_{c}-a_{j}^{(n)} \varepsilon-\tau^{(n)}\right)+n(t)
$$

$\tau^{(n)}$ represents the time delay of the pulse of the $n$-th device. We assume that reference transmitter and receiver are perfectly synchronized, i.e., $\tau^{(1)}=0$.

Delays $\tau^{(n)}$ with $n \neq 0$ are assumed independent and uniformly distributed random variables between 0 and Ts. The soft decision receiver output can be express in the following:

$Z=\int_{0}^{T b} r(t) m(t) d t$

where $m(t)$ is correlated mask, its expression as:

$$
m(t)=\sum_{j=0}^{N s-1} v\left(t-j T_{s}-C_{j}^{(1)} T_{s}\right)
$$

Where

$$
v(t)=P_{0}(t)-P_{0}(t-\varepsilon)
$$

The receiver estimates the bit according to all signals contribution:

$$
Z=Z_{u}+Z_{m u i}+Z_{n}
$$

where $Z_{u}, Z_{m u i}$ and $Z_{n}$ represent the useful signal, the MUI noise and thermal noise, respectively.

For orthogonal and the best binary PPM modulation, we use ML decision rule

$\left\{\begin{aligned} Z>0 & \Rightarrow \hat{a}=0 \\ Z<0 & \Rightarrow \hat{a}=1\end{aligned}\right.$ 
According to the ML decision rule, we can get the BER expression as following:

$$
\begin{aligned}
B E R & =\frac{1}{2} \operatorname{Pr}(\hat{a}=1 \mathrm{a}=0)+\frac{1}{2} \operatorname{Pr}(\hat{a}=1 \mid \mathrm{a}=0) \\
& =\operatorname{Pr}(\hat{a}=1 \mid \mathrm{a}=0) \\
& =\operatorname{Pr}(Z=1 \mid \mathrm{a}=0) .
\end{aligned}
$$

Assuming that $Z_{m u i}$ and $Z_{n}$ are zero-mean Gaussian random processes characterized by variance $\sigma_{m u i}^{2}$ and $\sigma_{n}^{2}$, respectively. Based in that works, for the binary PPM modulation the bit error probability is written by [2-3].

$$
\operatorname{Pr}_{b}=\frac{1}{2} \operatorname{erfc}\left(\sqrt{\frac{S I N R}{2}}\right)
$$

where SINR is the Signal-to-Interference and Noise Ratio and accounts for the useful, thermal and interference noise contributions. The useful signal contribution is the bit energy $E_{b}$ over the time of the bit and within the SGA assumption the following expression is obtained:

$$
\begin{aligned}
& \operatorname{SINR}=\frac{E_{b}}{\sigma_{n}^{2}+\sigma_{m u i}^{2}} \\
& \operatorname{SINR}=\left((S N R)^{-1}+(S I R)^{-1}\right)^{-1}= \\
& =\left(\left(\frac{E_{b}}{\sigma_{n}^{2}}\right)^{-1}+\left(\frac{E_{b}}{\sigma_{m u i}^{2}}\right)^{-1}\right)^{-1}
\end{aligned}
$$

where SNR and SIR represents the signal to thermal noise ratio, and the signal to MUI noise ratio respectively. If the signal energy can be looked as the sum of Ns pulses energy (per bit), the $\mathrm{E}_{\mathrm{b}}$ can be defined as:

$$
\begin{aligned}
E_{b} & =\left(Z_{u}^{2}\right) \\
& =\left(\sqrt{E_{R X}^{(1)}} \sum_{j=0}^{N_{s-1}} \int_{j T_{s}-C_{j}^{(1)} T_{c}}^{j T_{s}-C_{j}^{(1)} T_{c}+T_{c}} p_{0}\left(t-j T_{s}-C_{j}^{(1)} T_{c}\right) v\left(t-j T_{s}-C_{j}^{(1)} T_{c}\right) d t\right)^{2} \\
& =E_{R X}^{(1)}\left(N_{s} \int_{0}^{T_{c}} p_{0}(t)\left(p_{0}(t)-p_{0}(t-\varepsilon)\right) d t\right)^{2} \\
& =E_{R X}^{(1)} N_{s}^{2}\left(\int_{0}^{T_{c}} p_{0}(t) p_{0}(t) d t-\int_{0}^{T_{c}} d_{j}^{1}\left(p_{0}(t) p_{0}(t-\varepsilon)\right) d t\right)^{2} \\
E_{b} & =E_{R X}^{(1)} N_{s}^{2}\left(1-R_{0}(\varepsilon)\right)^{2} .
\end{aligned}
$$

Where $\mathrm{R}_{0}(\varepsilon)$ is the Autocorrelation function of $\mathrm{P}_{0}(\mathrm{t})$ At the binary PPM receiver output, the Variance of thermal noise is given bay:

$$
\sigma_{n}^{2}=N_{s} N_{0}\left(1-R_{0}(\varepsilon)\right)
$$

Expression for the SNR is then obtained:

$$
S N R_{n}=\frac{N_{s} E_{R X}^{(1)}}{N_{0}}\left(1-R_{0}(\varepsilon)\right)
$$

The features of MUI interference to the UWB receiver output can be defined as:

$$
m u i_{p}^{(n)}\left(\tau^{(n)}\right)=\sqrt{E_{R X}^{(n)}} \int_{0}^{2 T_{m}} p_{0}\left(t-\tau^{(n)}\right) v(t) d t
$$

where $2 T_{m}$ represents the duration of the PPM mask. $E^{(n)}$ is the energy received over each single pulse for the $\mathrm{n}^{\text {th }}$ interfering user. Assuming the delay $\tau^{n}$ is uniformly distributed over $[0, \mathrm{Ts}]$, the variance of the interfering noise is given by:

$$
\begin{aligned}
\sigma_{m u i_{p}^{n}}^{2} & =\frac{1}{T_{s}} \int_{0}^{T_{s}}\left(\sqrt{E_{R X}^{(n)}} \int_{0}^{2 T_{M}} p_{0}\left(t-\tau^{(n)}\right) v(t) d t\right)^{2} d \tau^{(n)} \\
& =\frac{E_{R X}^{(n)}}{T_{s}} \int_{0}^{T_{s}}\left(\int_{0}^{2 T_{M}} p_{0}\left(t-\tau^{(n)}\right) v(t) d t\right)^{2} d \tau^{(n)}
\end{aligned}
$$

If the time delay obeys the same distribution, can be written as:

$E_{m u i}=\frac{N_{s}}{T_{s}} \sigma_{M}^{2} \sum_{n=2}^{N u} E_{R X}^{(n)}$.

Where once again $\tau$ obeys the uniform distribution from zero to Ts and the $\sigma_{M}^{2}$ expressed as:

$$
\sigma_{M}^{2}=2 \int_{-T_{M}}^{2 T_{M}}\left(R_{0}(t)\right)^{2} d \tau
$$

Also, the signal to interference ratio (SIR) can be written as following:

$$
\begin{gathered}
S I R=\frac{E_{R X} N_{s}^{2}(1-R(\varepsilon))^{2}}{\frac{N_{s}}{T_{s}} \sigma_{M}^{2} \sum_{N=2}^{N u} E_{R X}^{(n)}} \\
S I R=\frac{(1-R(\varepsilon))^{2}}{\sigma_{M}^{2}} \frac{N_{s} T_{s}}{\sum_{N=2}^{N u} \frac{E_{R X}^{(n)}}{E_{R X}^{(1)}}}
\end{gathered}
$$




$$
\operatorname{SIR}=\frac{(1-R(\varepsilon))^{2}}{\sigma_{M}^{2}} \frac{N_{s} T_{s}}{\sum_{N=2}^{N u} \frac{E_{R X}^{(n)}}{E_{R X}^{(1)}}}
$$

The BER for 2PPM-TH-UWB system based on the SGA is thus [2-3-7]

$B E R=\frac{1}{2} \operatorname{erfc} \sqrt{\frac{\left(\left(\frac{E_{b}^{(1)}\left(1-R_{0}(\varepsilon)\right)}{N_{0}}\right)^{-1}+\left(\frac{\left(1-R_{0}(\varepsilon)\right)^{2}}{\left.\left.R_{b} \sigma_{M}^{2} \sum_{N=2}^{N_{u}} \frac{E_{R X}^{(n)}}{E_{R X}^{(1)}}\right)^{-1}\right)^{-1}}\right.\right.}{2}}$

Equation (18) shows that SNRn is maximum (BER is minimum) when autocorrelation $R_{0}$ is minimum, and can be maximized by selecting an optimal shift PPM value. Not that for orthogonal waveform $\left(\varepsilon>T_{m}\right), R_{0}(\varepsilon)=0$, than $1-R_{0}(\varepsilon)=1$. The optimum shift value, however, be smaller than $\mathrm{T}_{\mathrm{m}}$ if $R_{0}(\varepsilon)$ takes on negative value, that is $1-R_{0}(\varepsilon)>1$.

The optimal PPM-Shift varies with bit Rate $\mathrm{Rb}$ as well as the ordre of derivative of Gaussian pulse, his can be clearly seen in Figure 2 and Figure 3.

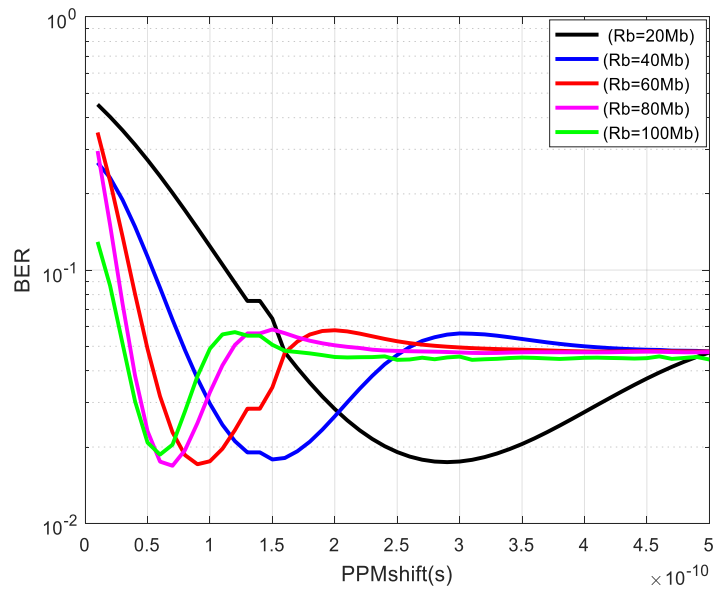

Fig. 2. BER of TH-UWB as a function of PPM-Shift for second derivative Gaussian pulse

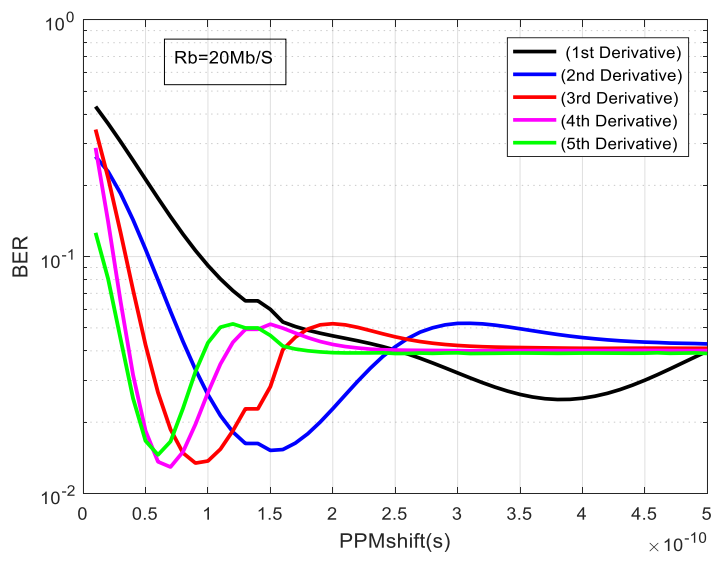

Fig. 3. BER of TH-UWB as a function of PPM-Shift for $R b=20 \mathrm{Mb}(\mathrm{b})$

\section{Proposed model}

Artificial Neural Networks (ANNs) model is an information processing paradigm that is inspired by the way biological unison to solve specific problem [8]. ANNs like people learn by experience not from programming, they are trained (learned) by repeatedly presenting examples (data) to the network which has a training rule and a weighted connection neuron that are adjusted on the basis of data that cannot be altered after the training, these weights help the network to make the decision without the need to use any other resource in decision-making.

In this work, ANN with Levenberg-Marquardt Learning Algorithm (LM) is used to optimize PPM-Shift for the THUWB under SGA. This optimal PPM-Shift is a function of the order of the derivative $(\mathrm{K})$ and the bit Rate $(\mathrm{Rb})$, this last is a function of the pulse duration (Tm) and the shaping factor for the pulse (tau), and the order of the derivative of Gaussian pulse(K) Figure 4. This function can be described by:

Optimal PPM-Shift $=f(\mathrm{~K}, \mathrm{Rb})$,

where PPM-shift is the values of PPM-shift optimized by the proposed approach and $f$ is a nonlinear function which maps the PPM-shift with the inputs parameters.

This network was trained with Levenberg-Marquardt backpropagation algorithm (trainlm). This network was chosen due to its good characteristic for solving fitting problems. The neural network must map well between a data set of numeric inputs and a set of numeric targets.

In training, the number of hidden layers, the number of the neurons in the hidden layers and Marquardt parameters were determined after trying various network structures, The network model used is a three-layer feed-forward network as illustrated in figure.4, two-layer feed-forward network with sigmoid hidden neurons and onelayer feed-forward network with linear output neurons, After a few experimental run, the number of neurons in the hidden layers was set to 20 neurons for each layer, [9-10].

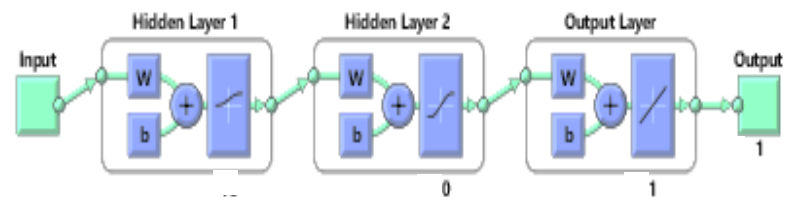

Fig. 4. Neural Network Training

The experimental data were to be separated into training, testing and validation data. There were no general guidelines available which could be followed to measure the ratio between the amount of training and testing and validation samples.

As per the recommended ratio of training, testing and validation, we used $70 \%$ data for training $15 \%$ for testing and $15 \%$ for validation.

\section{Performance Evaluation of ANN Training Algorithm}

The high correlation coefficient $(\mathrm{R}=0.99813)$ indicates that the PPMshift optimized by the LM-ANN perfectly fit (at $99.813 \%$ ) those computed by Autocorrelation method, figure (6).

In this work, the validation of the proposed estimator is 
evaluated by comparing its computed PPM-Shift values $\left(\varepsilon_{i}\right)$ with that found by the Autocorrelation method $\left(\varepsilon_{i}^{*}\right)$ using the root mean square error (RMSE), This indicator is calculated as:

$$
R M S E=\sqrt{\frac{1}{n} \sum_{i=1}^{n}\left(\varepsilon_{i}-\varepsilon_{i}^{*}\right)^{2}}
$$

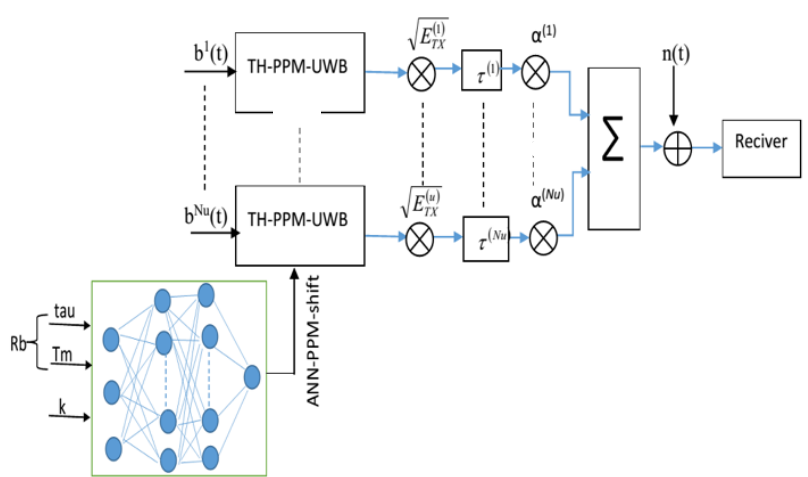

Fig. 5. New model for of TH-UWB with PPM modulation and ANN PPMshift

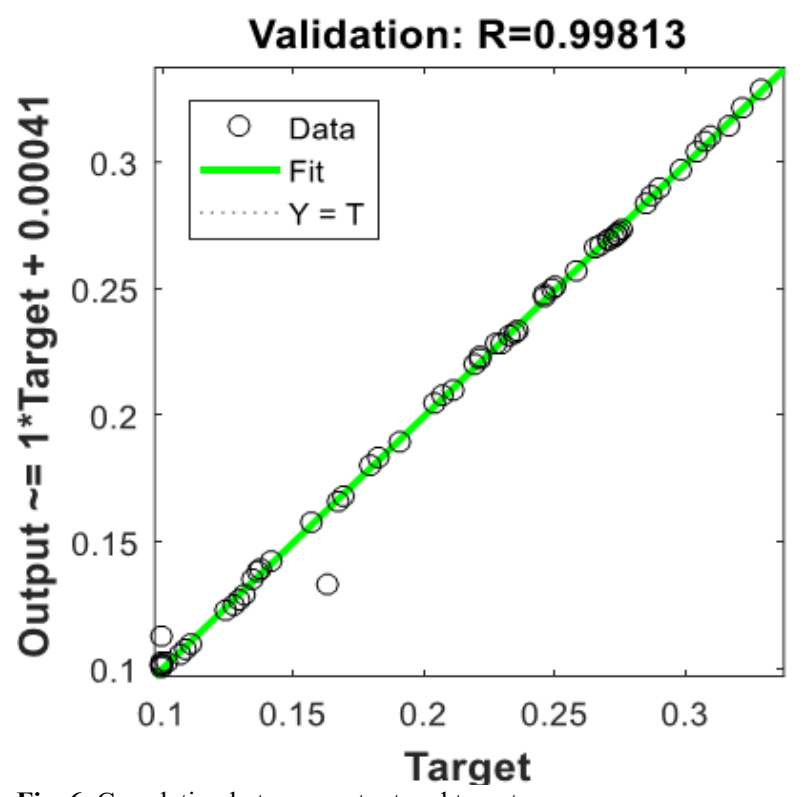

Fig. 6. Correlation between output and target

\section{Simulation, Results and discussion}

In the simulation, we assume that the collision happens when the waveform overlapping in MUI system. Now we consider a common situation: this is $\mathrm{Nu}$ asynchronous user binary PPM Time-Hopping system which uses the common interface to send binary data. It is also assumed that 3 pulses are used to transmit one bit $(N s=3)$ and the pulse repeat period Ts $=6 n s$. Each period Ts is divided into time slots (Tc) the transmit rate $(R b)$ will be variants with basic rate $R 0=20 \mathrm{MB} / \mathrm{s}$. The basic waveform is first 05 derivative of Gaussian pulse.

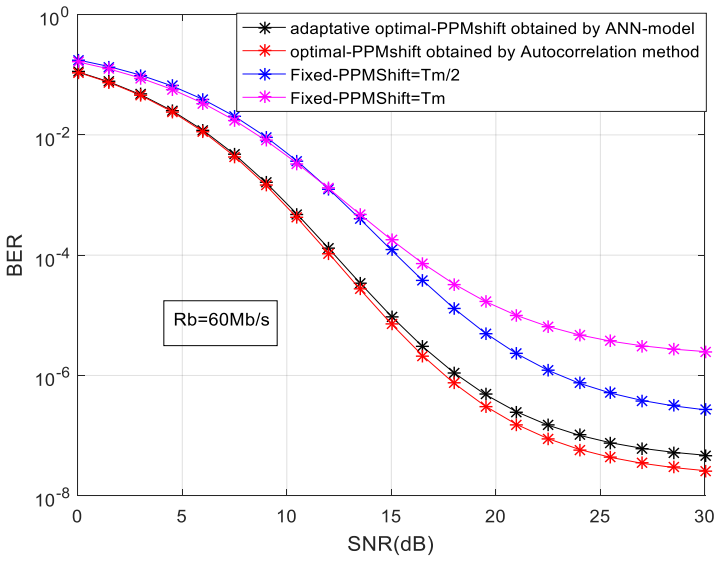

Fig. 7. BER of 2PPM-TH-UWB for different PPM-Shift

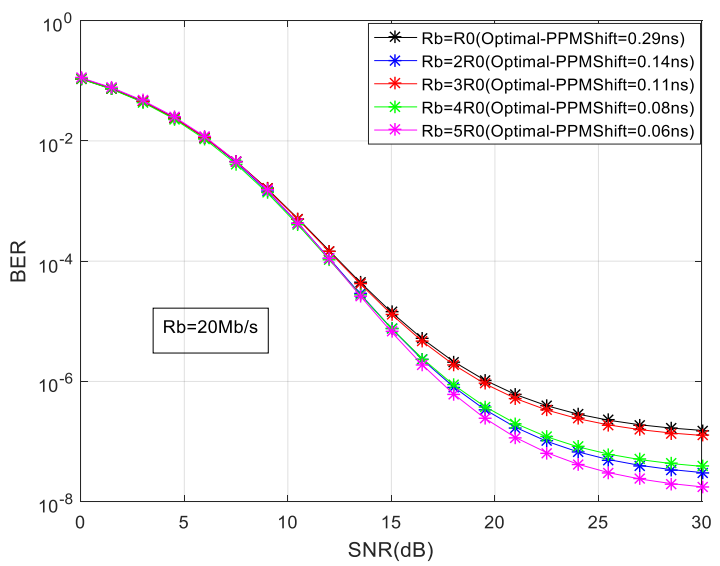

Fig. 8. BER of $2 P P M-T H-U W B$ for variable data Rate with optimal PPM-Shift

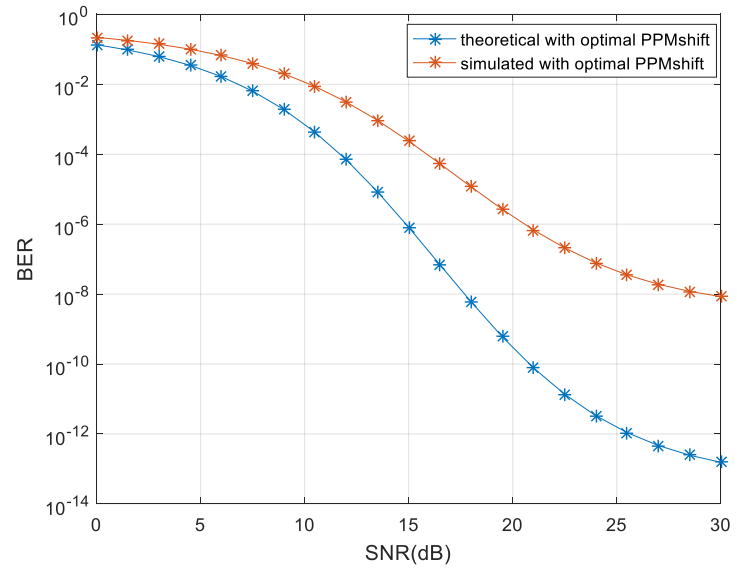

Fig. 9. Theoretical and simulated BER of $2 P P M-T H-U W B$ versus the SNR with optimal PPM-Shift obtained by ANN approach

The variation of the error bit error Rate of the adaptive optimal PPM-Shift with respect to the SNR values is shown in figure 7 and 8 . We also showed the variation of the BER for two fixed PPM-Shift cases (PPM- Shift $=\mathrm{Tm} / 2$, and Tm), which are the most frequently PPM-Shift used in our system. From this results, we conclude that BER of the adaptive optimal PPM-Shift is better than those of the fixed case and gives almost the same performance as the Autocorrelation method,

As expected, the performance of the proposed model with the optimal adaptive PPM-Shift calculator reacts positively with the $\mathrm{Rb}$ values.

Figure 9 compares theoretical and measured BER for 2PPM-TH-UWB with optimal PPM-Shift obtained by ANN 
approach, this figure shows a good agreement between theoretical performance and simulation results.

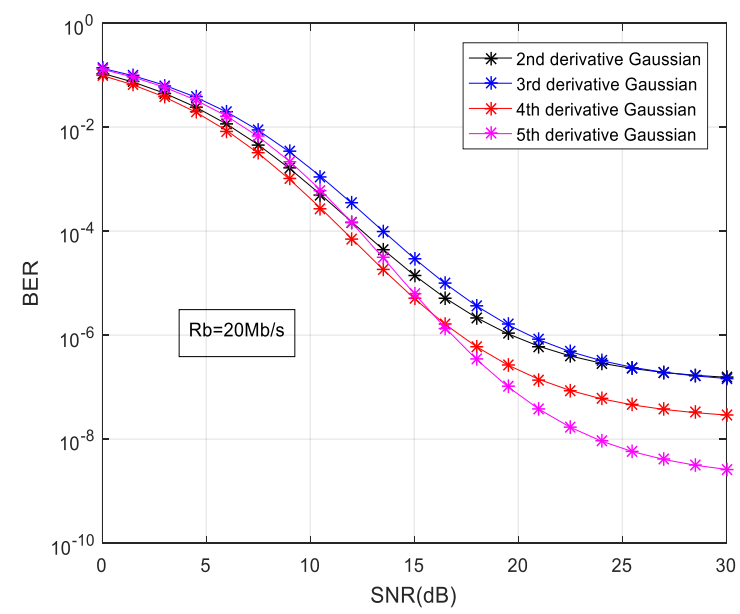

(a)

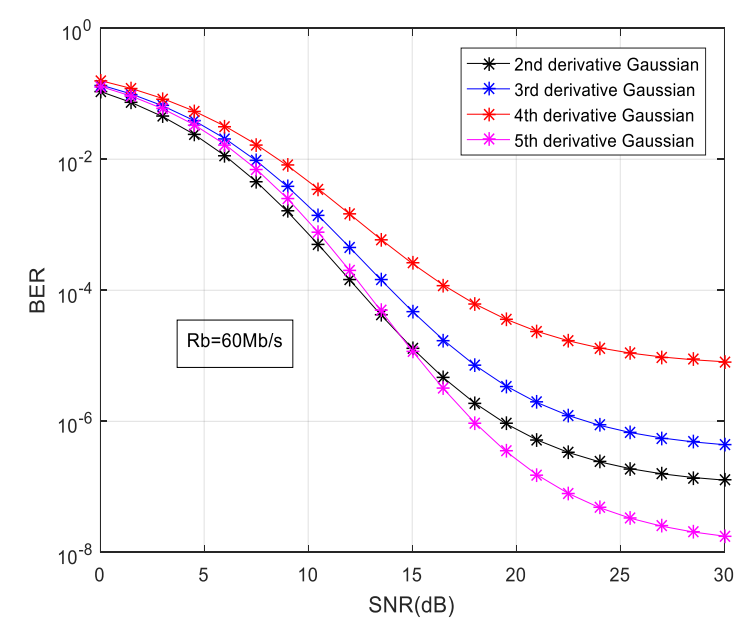

(b)

Fig. 10. BER performance of different pulses with optimal PPM-Shift for $\mathrm{Rb}=20 \mathrm{Mb} / \mathrm{s}$ (case $\mathrm{a}$ ), for $\mathrm{Rb}=60 \mathrm{Mb} / \mathrm{s}$ (case $b$ ).
In figure 10, with optimum value of PPM-Shift, The best results are obtained with the $5^{\text {th }}$ and the 4th derivative of Gaussian pulse for $\mathrm{Rb}=20 \mathrm{Mb} / \mathrm{s}$ (case a), but the the $5^{\text {th }}$ and the 2 nd derivative of Gaussian pulse gives low value of BER for $\mathrm{Rb}=60 \mathrm{Mb} / \mathrm{s}$ (case b), this justifies the choice of the pulse depends heavily on the value of data Rate $\mathrm{Rb}$.

\section{Conclusions}

The precedent results indicate that the PPM-Shift directly affects the performance of 2PPM-TH-UWB systems. It is clear that our systems will achieve the best performance when PPM-Shift is optimal, but sometimes this value is very small, for this difficult to implement.

In this paper, a new adaptive optimal PPM-Shift estimator based on LM-ANN for the 2PMM-TH-UWB and PPM modulation is proposed. The performance of the model using the proposed PPM-Shift estimator has been compared with a simulated TH-UWB that uses a fixed value of PPMShift and a theoretical TH-UWB with optimal PPM-Shift computed using the Autocorrelation method. The obtained results show that the proposed model clearly very compatible with multi-Rate system in terms of probability of error. This work is still ongoing and further work is planned to investigate other artificial models, like SVM, RBF and ANFIS.

\section{Acknowledgements}

We wish to thank all the professors of the Faculty of Electrical Engineering Djillali Liabes University, for the comments and suggestions that have helped to improve this work.

This is an Open Access article distributed under the terms of the Creative Commons Attribution License

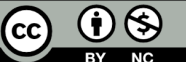

\section{References}

1. Djamel Abed, Salah Redadaa, and Smail Tedjini, "Closed-form pdf for multiuser tr-uwb Systems under Gaussian noise and impulsive interference," Progress in Electromagnetics Research C, Vol. 40, $175-186,2013$.

2. A.Maaref, S. Elahmar, and M. Bouziani, "Performance Analysis of PC and SGA models for UWB using Modified Hermite," IEEE Xplore: 21 March 2013 DOI: 10.1109/SETIT.2012.6481972.

3. Huibin Xu, Lijie Zhou, "Analysis of the BER of multi-user IR-UWB system Based on the SGA, Applied Mechanics and Materials," Vols 71-78 (2011) pp 4786-4789 (C) (2011) Trans Tech Publications,Switzerland doi:10.4028/www.scientific.net/AMM.7178.4786

4. Himanshu B. Soni, U.B. Desai and S.N. Merchant, "Low complexity RAKE receiver for TH-based multiuser UWB system with realistic UWB indoor channel," Int. J. Ultra Wideband Communications and Systems, Vol. 2, No. 1, 2011

5. V. Yajnanarayana, S. Dwivedi, A. Angelis, P. Handel, "Design of impulse radio UWB transmitter for short range communications using PPM signals," IEEE CONECCT 2013.
6. Vikas Goyal B.S Dhaliwal, "Improving ultra wideband (uwb) system by modified random combination of pulses," Engineering Review, Vol. 38, Issue 2, 189-203, 2018.

7. D. Feng, S.Ghauri, and Q.Zhu, "Application of the MUI Model Based on Packets Collision (PC) in UWB Ad-hoc Network" Proceedings of the 2009 IEEE international Conference on D.

8. Sonali B. Wankhede, "Analytical Study of Neural Network Techniques: SOM, MLP and Classifier-A Survey," IOSR Journal of Computer Engineering (IOSR-JCE) e-ISSN: 2278-0661, p- ISSN: 2278-8727Volume 16, Issue 3, Ver. VII (May-Jun. 2014), PP 86-9.

9. P. Bhuvaneswari, Ramesh.G.P, "Levenberg-marquardt algorithm to identify the fault analysis for industrial applications," International Journal of Engineering and Technology, 7 (1.2) (2018) 141-150.

10. Kuryati Kipli, Mohd Saufee Muhammad, Sh. Masniah Wan Masra, Nurdiani Zamhari, Kasumawati Lias, Dayang Azra Awang Mat, "Performance of Levenberg-Marquardt Backpropagation for Full Reference Hybrid Image Quality Metrics," Proceedings of the International MultiConference of Engineers and Computer Scientists 2012 IMECS 2012, 14-16 March, 2012, Hong Kong. 